\title{
Evolutionary Generation of Implicative Fuzzy Rules for Design Knowledge Representation
}

\author{
Mark Freischlad, Martina Schnellenbach-Held, and Torben Pullmann \\ University of Duisburg-Essen, Institute of Structural Concrete, \\ Universitätsstraße 15, 45141 Essen, Germany \\ \{mark.freischlad, m.schnellenbach-held, \\ torben.pullmann\} @uni-due.de
}

\begin{abstract}
In knowledge representation by fuzzy rule based systems two reasoning mechanisms can be distinguished: conjunction-based and implication-based inference. Both approaches have complementary advantages and drawbacks depending on the structure of the knowledge that should be represented. Implicative rule bases are less sensitive to incompleteness of knowledge. However, implication-based inference has not been widely used. This disregard is probably due to the lack of suitable methods for the automated acquisition of implicative fuzzy rules. In this paper a genetic programming based approach for the data-driven extraction of implicative fuzzy rules is presented. The proposed algorithm has been applied to the acquisition of rule bases for the design of reinforced concrete structural members. Finally an outlook on the application of the presented approach within a machine learning environment for evolutionary design and optimization of complex structural systems is given.
\end{abstract}

\section{Introduction}

The preliminary structural design process is a knowledge intensive task. Knowledge based systems are suitable to support engineers within this process. These systems offer the possibility to represent knowledge in a transparent and comprehensible way. In order to handle the complexity, incompleteness and vagueness of experience knowledge, fuzzy reasoning mechanisms are of special interest [1].

In the following section the fundamentals of implicative rule bases as well as the differences between conjunctive-based and implicative-based inference are presented. In section 3 a new genetic programming based approach for the data-driven generation of implicative fuzzy rule bases is shown. The developed algorithm has been applied to the acquisition of rule bases for the design of reinforced concrete structural members. The results of these tests are demonstrated in section 4. An outlook on the application of the presented approach within a machine learning environment for evolutionary design and optimization of complex structural systems and concluding remarks is given in section 5. Conclusions and a short outlook are presented in the last section. 


\section{Implicative Fuzzy Rule Bases}

Two basic types of fuzzy rules can be distinguished [2]. A rule "If $X$ is $A_{\mathrm{i}}$, then $Y$ is $B_{\mathrm{i}}$ " can be interpreted as a mapping of the domain $U(X \in U)$ to the domain $V(Y \in V)$. In this case each rule of a rule base encodes possible values for $Y$. This semantic is represented by using a conjunctive implication operator, e.g. the minimum or the product operator. As each conjunctive fuzzy rule defines a possible fuzzy point and a set of conjunctive fuzzy rules defines a fuzzy graph, rules have to be combined disjunctively. The aggregation of two (crisp) conjunctive rules is illustrated in figure 1a.

Furthermore, the rule can be interpreted as a logic implication. In this case the rule excludes impossible values of $V$. Consequential the implication operator has to satisfy the equivalence of the implication $A_{\mathrm{i}} \rightarrow B_{\mathrm{i}}$ and the proposition $\neg A_{\mathrm{i}} \vee B_{\mathrm{i}}$, e.g. the Kleene/Dienes or the Lukasiewicz operator. An implicative fuzzy rule base (FRB) represents a set of fuzzy constraints on the values of $Y$. Hence the rules have to be combined conjunctively. Figure $1 \mathrm{~b}$ shows the aggregation of two implicative rules.
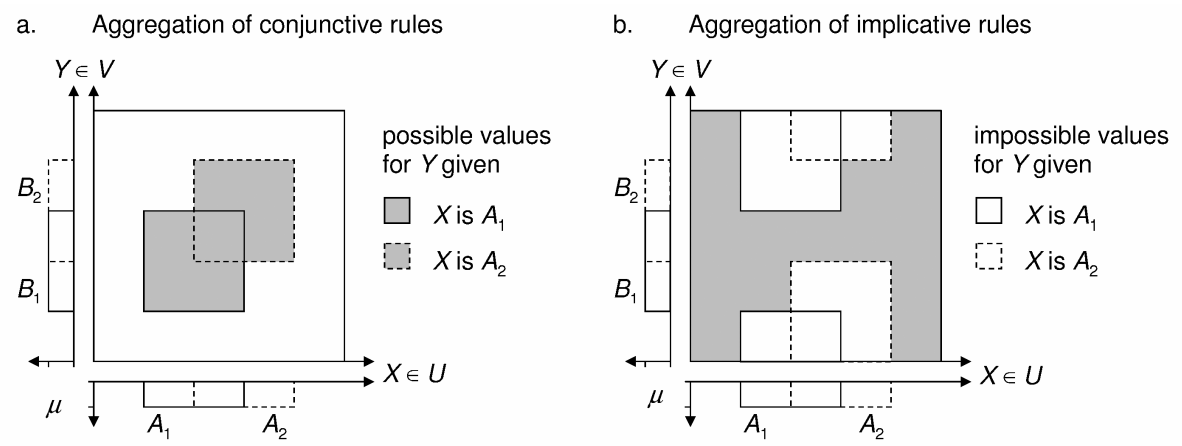

Fig. 1. Aggregation of (a) conjunctive rules and (b) implicative rules

The output fuzzy set of a conjunctive fuzzy rule base is the set of all possible values for $Y$ given the situation $X$. By means of defuzzification a crisp value for $Y$ can be derived. The output fuzzy set of an implicative fuzzy rule base represents an upper bound of possible values on $V$, according to the knowledge considered within the rule base. The determination of a crisp output value by means of defuzzification is not suitable in general. Often the incorporation of further knowledge is required. However, in combination with subsequent search mechanisms the elimination of impossible values can be very useful.

Within the scope of knowledge based decision support different areas for the application of the two types of rules bases can be stated. Depending on the structure of the knowledge to be represented the two reasoning mechanisms hold advantages and disadvantages. One advantage of conjunctive fuzzy rule bases is the possibility to directly obtain a crisp output value. To ensure the reliability a complete rule base and the consideration of all influence parameters is necessary. For the support of more complex decisions implicative rule bases are of advantage. In this case, not all influence parameters have to be considered in the rule base. On the one hand this leads to a 
reduced specificity of output sets. On the other hand the size of the rule base is reduced significantly. Hence the interpretability is increased.

\section{Evolutionary Learning of Implicative Fuzzy Rules}

In recent years many approaches for the data-driven generation of conjunctive fuzzy rule bases have been proposed, e.g. neuro-fuzzy systems and genetic fuzzy systems [3]. In former works the authors have presented a genetic programming based algorithm for the multi-objective optimization of fuzzy systems [4]. The optimization process is guided by the demands on the accuracy and the interpretability of fuzzy systems. Based on this approach the Rule Base Extraction and Maintenance (REM) algorithm has been developed. The REM algorithm serves for the generation and optimization of both, conjunctive and implicative FRBs.

As pointed out in the previous section, the output fuzzy sets of both types of FRBs are interpreted in a different manner. Thus, the evaluation of the quality differs. In case of conjunctive FRBs a major measure of quality is the approximation error of the defuzzified outputs and the training data. Further measures have been proposed, e.g. the completeness of the rule base [5]. For the evaluation of implicative FRBs three measures are proposed: the specificity of the output fuzzy sets, the consistency of the output values of case examples with the corresponding output fuzzy sets and the congruency of the input space region covered by the case base with the region covered by the rule base.

Given a set of $N_{C B}$ case examples (case base), where each case is represented by a set of $M$ input variables and one output variable. Assume an implicative fuzzy rule base, consisting of $N_{R}$ rules is to be evaluated.

\subsection{Specificity}

A major purpose of an implicative FRB is the elimination of impossible values of the output variable. A measure for this purpose is the mean specificity of the output fuzzy sets on the input vectors of the case base. The specificity is defined by

$$
\mathrm{F}_{\mathrm{SP}}=1-\sum_{\mathrm{i}=1}^{\mathrm{N}_{\mathrm{CB}}}\left(\int \mu_{\mathrm{i}}(\mathrm{y}) \mathrm{dy} / \int \mathrm{dy}\right) / \mathrm{N}_{\mathrm{CB}}
$$

where $\mu_{\mathrm{i}}(\mathrm{y})$ is the membership function of the output fuzzy set on the $i$ th case example. The higher the specificity, the more values of the output variable are (partly) excluded and consequently the more valuable is the rule base in terms of finding a crisp output value.

\subsection{Consistency}

By increasing the specificity the rule base might get inconsistent with the case knowledge. That means the output value of a case example is determined as an (highly) impossible value. The mean consistency of the rule base with the underlying case knowledge is derived by 


$$
\mathrm{F}_{\mathrm{CS}}=\sum_{\mathrm{i}=1}^{\mathrm{N}_{\mathrm{CB}}} \mu_{\mathrm{i}}\left(\mathrm{y}_{\mathrm{i}}\right) / \mathrm{N}_{\mathrm{CB}}
$$

where $y_{\mathrm{i}}$ is the output value of the $i$ th case example and $\mu_{\mathrm{i}}\left(\mathrm{y}_{\mathrm{i}}\right)$ is the corresponding degree of membership to the output fuzzy set on case $i$.

\subsection{Congruency}

An implicative FRB should only fire in those areas of the input domain that are covered by the knowledge contained in the case base. In order to determine the congruency two parameters, the fuzzy coverage of the input domain $\bar{U}(\bar{x} \in \bar{U})$ by the case base $\mu_{\mathrm{CB}}(\overline{\mathrm{x}})$ and the coverage by the rule base $\mu_{\mathrm{RB}}(\overline{\mathrm{x}})$ are defined. The coverage of $\overline{\mathrm{U}}$ by the case base is derived by

$$
\mu_{\mathrm{CB}}(\overline{\mathrm{x}})=\max \left(\mu_{\mathrm{C}, \mathrm{i}}(\overline{\mathrm{x}})\right), \quad \mathrm{i}=1,2, \ldots, \mathrm{N}_{\mathrm{CB}}
$$

with

$$
\mu_{\mathrm{C}, \mathrm{i}}(\overline{\mathrm{x}})=\prod_{\mathrm{m}=1}^{\mathrm{M}} \mathrm{e}^{-\left(\frac{\mathrm{x}_{\mathrm{m}}-\mathrm{x}_{\mathrm{m}, \mathrm{i}}}{\sigma_{\mathrm{m}}}\right)^{2}}
$$

and with $x_{\mathrm{m}, \mathrm{i}}$ is the value of the $m$ th input variable of case $i$ and $\sigma_{\mathrm{m}}$ is the predefined fuzziness of input variable $m$. By means of this fuzziness the degree of coverage in the neighbourhood of a case can be adapted.

The coverage of $\bar{U}$ by the rule base is derived by

$$
\mu_{R B}(\bar{x})=\max \left(\mu_{R, j}(\bar{x})\right), \quad j=1,2, \ldots, N_{R}
$$

where $\mu_{R, j}(\bar{x})$ is the degree of confidence of rule $\mathrm{j}$.

The congruency measure is defined by

$$
\mathrm{F}_{\mathrm{CG}}=1-\int \max \left(0, \mu_{\mathrm{RB}}(\overline{\mathrm{x}})-\mu_{\mathrm{CB}}(\overline{\mathrm{x}})\right) \mathrm{d} \overline{\mathrm{x}} / \int \mu_{\mathrm{RB}}(\overline{\mathrm{x}}) \mathrm{d} \overline{\mathrm{x}}
$$

The overall accuracy fitness $F_{\mathrm{AC}}$ of the rule base is composed of the presented measures:

$$
\mathrm{F}_{\mathrm{AC}}=\left(\mathrm{a}_{\mathrm{SP}} \cdot \mathrm{F}_{\mathrm{SP}}+\mathrm{a}_{\mathrm{CS}} \cdot \mathrm{F}_{\mathrm{CS}}+\mathrm{a}_{\mathrm{CG}} \cdot \mathrm{F}_{\mathrm{CG}}\right) /\left(\mathrm{a}_{\mathrm{SP}}+\mathrm{a}_{\mathrm{CS}}+\mathrm{a}_{\mathrm{CG}}\right)
$$

By means of the weight parameters $a_{\mathrm{SP}}$ (specificity), $a_{\mathrm{CS}}$ (consistency) and $a_{\mathrm{CG}}$ (congruency) the optimization process can be adapted in order to increase a preferred quality.

Besides this objective the REM algorithm takes into account the demands on the interpretability of the rule base represented by the number of rules [4]. The overall fitness of an individual is determined by the pareto-rank based approach presented by Fonseca and Fleming [6]. 


\section{Evaluation}

The developed approach was evaluated by applying it to a real world data set for the determination of the beam height of a beam slab. The input variables are the span length of the beam $l_{\mathrm{b}}$, the span length of the slab $l_{\mathrm{s}}$ and the life load on the slab $q$. The output variable is the beam height $h_{\mathrm{b}}$.

To demonstrate the advantages of implicative FRBs the goal was to find a rule base considering only the major influence parameters for this design decision. In order to simulate knowledge incompleteness only case examples covering parts of the input domain were chosen. The fuzzy coverage by the case base is shown in figure $2 \mathrm{a}$.

The REM algorithm was run for 300 generations; the population size was set to 200 individuals. The maximum number of rules was set to 8 rules. The weight parameters of the accuracy fitness components were set to $a_{\mathrm{SP}}=a_{\mathrm{CS}}=a_{\mathrm{CG}}=1.0$.

The pareto-optimal rule base consisting of 6 rules is presented in further detail: Figure $2 b$ shows the coverage of the input domain by this rule base. It is obvious that any rule fires significantly outside the fuzzy support of the case base, confirmed by the value of the congruency measure $F_{\mathrm{CG}}=0.978$.
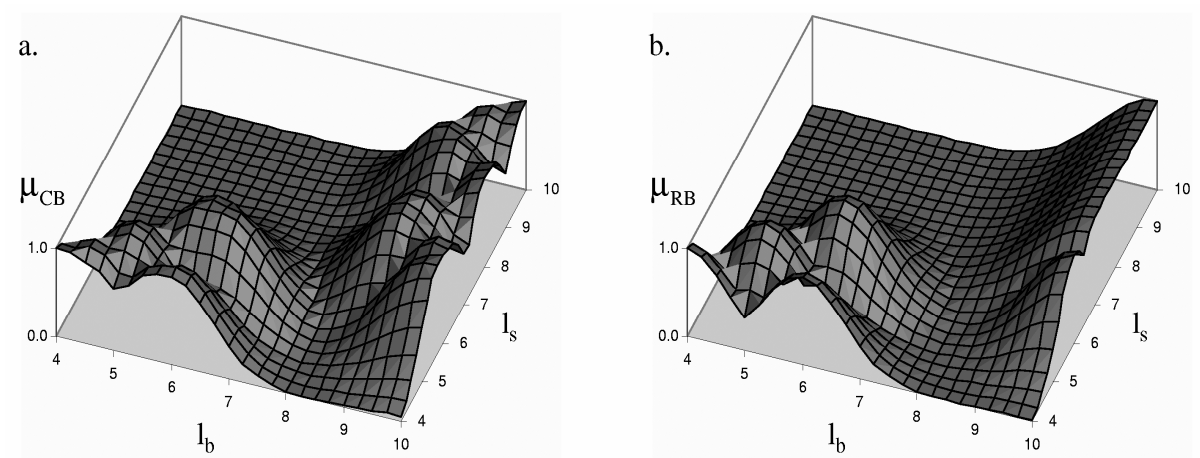

Fig. 2. Coverage of the input domain (a) by the case base and (b) by the rule base

Figure 3 shows the output fuzzy sets for three situations: $S_{1}\left(l_{\mathrm{b}}=5 \mathrm{~m}, l_{\mathrm{s}}=5 \mathrm{~m}\right)$, $S_{2}\left(l_{\mathrm{b}}=10 \mathrm{~m}, l_{\mathrm{s}}=7 \mathrm{~m}\right)$ and $S_{3}\left(l_{\mathrm{b}}=8 \mathrm{~m}, l_{\mathrm{s}}=6 \mathrm{~m}\right)$. For $S_{1}$ a highly specific fuzzy set is obtained (fig. 3a). This set is in accordance with the case examples $C_{1}\left(l_{\mathrm{b}}=5 \mathrm{~m}, l_{\mathrm{s}}=5\right.$ $\left.\mathrm{m}, q=3 \mathrm{kN} / \mathrm{m}^{2}, h_{\mathrm{b}}=35 \mathrm{~cm}\right)$ and $C_{2}\left(l_{\mathrm{b}}=5 \mathrm{~m}, l_{\mathrm{s}}=5 \mathrm{~m}, q=9 \mathrm{kN} / \mathrm{m}^{2}, h_{\mathrm{b}}=42 \mathrm{~cm}\right)$. The fuzzy set obtained for $S_{2}$ is less specific (fig. 3b), representing well the larger range of the optimal beam height $\left(h_{\mathrm{b}}=65 \sim 82 \mathrm{~cm}\right)$ for typical values of the life load $(q=$ $3 \sim 9 \mathrm{kN} / \mathrm{m}^{2}$ ).

For $S_{3}$ almost any value of $h_{\mathrm{b}}$ is ruled out (fig. $3 \mathrm{c}$ ). There was no case highly similar to this situation in the case base. Consequently almost any information can be provided and a nearly unrestricted search for a suitable output value has to be performed. Assumed a solution for this situation was found, the rule base can be extended based on this newly discovered knowledge.

The mean specificity for all case examples is $F_{\mathrm{SP}}=0.586$. The generated rule base is highly consistent with the underlying case base $\left(F_{\mathrm{CS}}=0.956\right)$. 

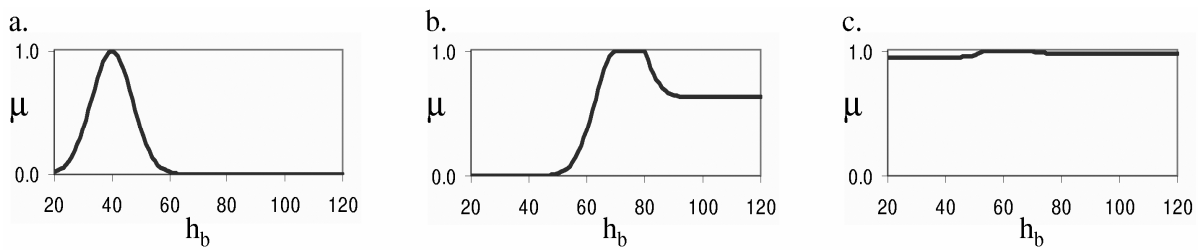

Fig. 3. Output fuzzy sets for (a) $l_{\mathrm{b}} / l_{\mathrm{s}}=5 \mathrm{~m} / 5 \mathrm{~m}$, (b) $l_{\mathrm{b}} / l_{\mathrm{s}}=10 \mathrm{~m} / 7 \mathrm{~m}$ and (c) $l_{\mathrm{b}} / l_{\mathrm{s}}=8 \mathrm{~m} / 6 \mathrm{~m}$

\section{A Machine Learning Environment for Structural Design}

The developed REM algorithm has been implemented into a machine learning environment for the conceptual design of concrete structures. Its core is a hybrid knowledge based evolutionary design system for the optimization of high-rise concrete structures. A prototype of this system has been presented in [7]. It was developed for the discrete optimization of the structural topology as well as the continuous optimization of single structural members. The structure is represented by a 3D product model based building information model. A finite element system for static and dynamic analyses of design candidates is included.

\subsection{Knowledge Augmented Design Optimization}

The conceptual design of high-rise structures includes major decisions on structural systems, e.g. the lateral load-bearing system, and subsidiary decisions on the dimension of structural members. In order to reduce the problem size two methods for the incorporation of design knowledge within the optimization process have been implemented.

Conjunctive fuzzy rule bases are used for direct determination of design variables. Thereby the number of optimization variables is decreased. Since the generation of reliable conjunctive fuzzy rule bases requires complete knowledge this method is restricted to secondary design decisions and ordinary conditions.

Highly complex design decisions are supported by implicative fuzzy rule bases. The search space of optimization variables is restricted according to the output fuzzy sets. Provided that knowledge is available for a given situation, the search space can be reduced significantly.

\subsection{Machine Learning of Design Knowledge}

In the scope of knowledge based systems the acquisition of knowledge by human experts is laborious and time-consuming. To overcome this problem the REM algorithm for the automated acquisition of design knowledge has been implemented into the presented design system. Figure 4 shows the architecture of the proposed machine learning environment.

The project data base contains the building information models of completed designs. When a new rule base is to be generated, the input and output variables have to be defined. Based on the project data base a set of case examples is retrieved. In the next step a fuzzy rule base is generated by the REM algorithm. The manual knowledge acquisition component serves human experts for checking and manipulation of 


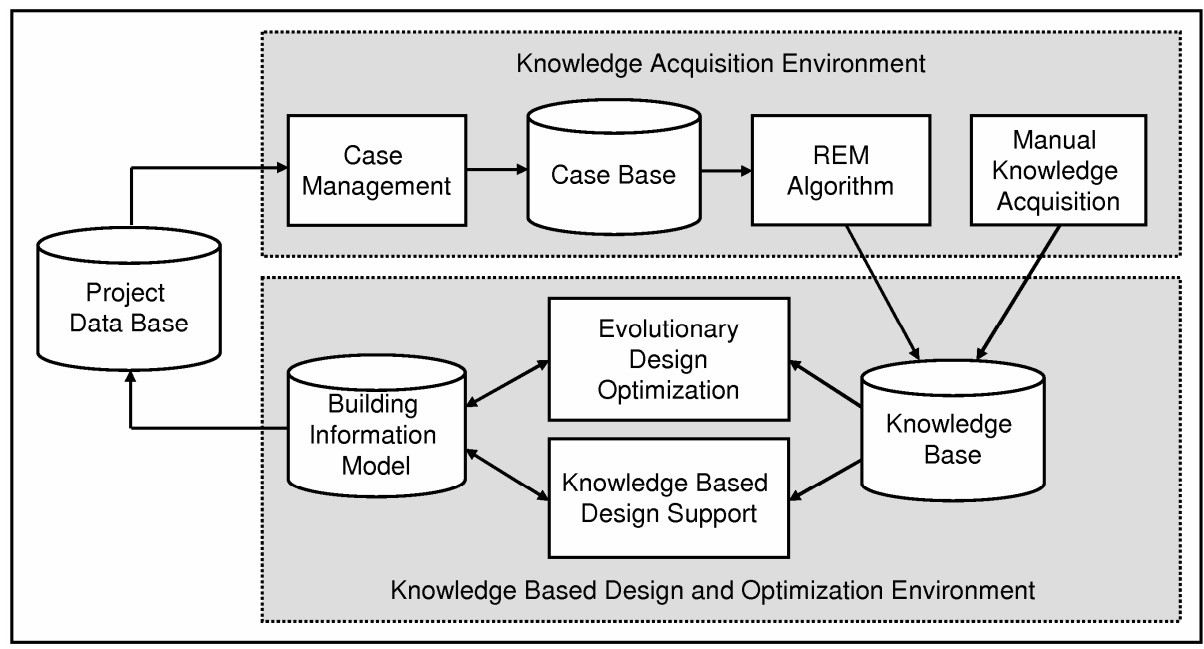

Fig. 4. Architecture of a machine learning environment for structural design

the knowledge base. Learned rule bases are incorporated within further optimization tasks as described in section 5.1.

Every time a project is completed, the project data base and, subsequently, the case base is updated. If necessary, the rule base is revised or extended.

\section{Conclusions}

In this paper a genetic programming based approach for the data-driven generation of implicative fuzzy rules was presented. Three measures for the evaluation of implicative fuzzy rule bases were proposed. The evaluation of the developed REM algorithm on a real world problem has shown that the generated fuzzy rule bases fulfill the demands on the specificity and the consistency with the knowledge of the underlying case base.

The application of REM within a machine learning environment for evolutionary design and optimization of complex structural systems was presented. Currently the authors investigate the impact of the proposed approach on the exploration of innovative design solutions.

\section{References}

1. Schnellenbach-Held, M., Freischlad, M.: Fuzzy Rule Based Models for Slab System Design. In: Schnellenbach-Held, M., Denk, H. (eds.): Advances in Intelligent Computing in Engineering. Proceedings of 9th International EG-ICE Workshop, VDI-Fortschritt-Berichte, Darmstadt (2002) 134-143

2. Dubois, D., Prade, H., Ughetto, L.: A new perspective on reasoning with fuzzy rules. International Journal of Intelligent Systems, V. 18 N. 5 (2003) 541-567 
3. Cordón, O., Gomide, F., Herrera, F., Hoffmann, F., and Magdalena, L.: Ten years of genetic fuzzy systems: current framework and new trends. Fuzzy Sets and Systems, Vol. 141, Issue 1 (2004) 5-31

4. Freischlad, M., Schnellenbach-Held, M.: A machine learning approach for the support of preliminary structural design. Advanced Engineering Informatics, Vol. 19, No 4 (2005) 281-287

5. Jin, Y., von Seelen, W., Sendhoff, B.: On Generating FC ${ }^{3}$ Fuzzy Rule Systems from Data Using Evolution Strategies. IEEE Transactions on Systems, Man and Cybernetics, Part B 29(4) (1999) 829-845

6. Fonseca, C. M., Fleming, P. J.: Genetic algorithms for multiobjective optimization: Formulation, discussion and generalization. In: Forrest, S. (ed.): Genetic Algorithms: Proceedings of the Fifth International Conference. San Mateo, CA: Morgan Kaufmann (1993) 416-423

7. Pullmann, T., Skolicki, Z., Freischlad, M., Arciszewski, T., De Jong, K., SchnellenbachHeld, M.: Structural Design of Reinforced Concrete Tall Buildings: Evolutionary Computation Approach Using Fuzzy Sets. In: Ciftcioglu, Ö., Dado, E. (eds.): Intelligent Computing in Engineering, Foundation of Design Research SOON (2003) 53-61 